\title{
Correction to "Abuse of Prescription and Over-the- Counter Medications"
}

In the abovementioned article (Lessenger JE, Feinberg SD.J Am Board Fam Med 2008;21:45-54), the institution affiliation information is incorrect. It should read: From private practice, Benicia, California (JEL), and the Department of Orthopaedic Surgery-Physical and Rehabilitation Medicine and the Department of Anesthesia-Pain Management Center, Stanford University School of Medicine, Stanford, California. We apologize for the error, and we regret any confusion or inconvenience it may have caused.

(doi: 10.3122/jabfm.2008.02.080005.) 